\title{
Interactive comment on "Spiciness theory revisited, with new views on neutral density, orthogonality and passiveness" by Rémi Tailleux
}

\section{Anonymous Referee \#1}

Received and published: 16 June 2020

This manuscript aims at clarifying the long-debated definition of a passive variable along neutral/isopycnal layers, commonly referred as "spiciness". The paper clarifies and demonstrates that the use of thermohaline anomalies (in particular absolute salinity) along neutral surfaces is sufficient to provide orthogonality in physical space. The long sought orthogonality in thermohaline space is showed to be flawed and not necessary to construct an inert variable along neutral surfaces. Moreover, the author discusses and resolves several issues raised by the definition of a physical variable satisfying the properties of spiciness. The existence of neutral surfaces is revealed to be key to the construction of a spiciness-like variable. By using theoretical arguments and a quasi-linear transformation of T/S space, the author also compares published definitions based on different assumptions and unifies them under basic principles. 
I found the manuscript very interesting and well written. It surely provides an important step forward to the study of water mass. I therefore only have a few minor comments and recommend this paper to be published.

160 and Fig 1 : What is the source of the data shown?

Fig2 : In caption : $\sin \left(\nabla \sigma_{1}, \nabla \xi\right)$ ? Why is the yellow histogram closer to 0 (ie sine closer to 1 , angle closer to $\pi / 2$ ), but described as the less orthogonal? Have the blue and yellow histograms been swapped? How does $\Theta$ variable compare to $S_{A}$ in terms of orthogonality?

1149 : What would be the proportion of the world ocean covered in that range? To identify regions where a spiciness definition would be challenged could be an interesting add to the paper.

- Along the manuscript, it is commonly referred to "orthogonality in physical space" and I think it would be nice to have a clear definition of what it means in introduction.

- I have the feeling that in regions of the ocean with temperature-driven density, salinity anomalies will have be a better choice to construct an inert variable. Am I speculating too much? Would $\Theta^{\prime}$ be any better than $S_{A}^{\prime}$ where density is salinity-driven (eg, coastal ocean, near sea-ice, Mediterranean, Red, Black Seas, ...)?

Interactive comment on Ocean Sci. Discuss., https://doi.org/10.5194/os-2020-39, 2020.
OSD

Interactive

comment 